\title{
Ueber das Verhältniss zwischen Reactionsvermögen und Concentration der Schwefelsäure;
}

\author{
von \\ Wilhelm Vaubel.
}

Es ist in neuerer Zeit üblich geworden, ganz allgemein die Reactionsfähigkeit von Elektrolyten auf das Vorhandensein der Jonen zu beziehen. Sicherlich gewinnt durch eine derartige Betrachtungsweise eine grosse Anzahl von Reactionen an Anschaulichkeit und Verständniss, und es würde wenig verständig erscheinen, wollte man einiger Auswüchse halber diese Art der Betrachtung chemischer Reaktionen durchaus verdammen. Immerhin muss jedoch hervorgehoben werden, dass ein Zuviel auch hier wie überall nur schädlich wirken kann. Der Zweck dieser Zeilen ist deshalb, darauf hinzuweisen, dass sicherlich neben der elektrolytischen Dissociation der reagirenden Körper auch noch andere Umstände in Betracht kommen, dass die Individualität sich nicht immer in das Schema hineinpressen lässt, sondern gar häufig die Schranken überspringt, die der ordnende Sinn für die Gesammtheit der Individuen gezogen hat.

Ein gutes Beispiel für die Behauptung, dass nicht gerade alle Reactionen als Jonenreactionen aufzufassen sind, bietet nun die Schwefelsäure. Es ist schon längere Zeit bekannt, dass die Schwefelsäure eine Reihe von Hydraten bildet, von denen einige isolirt werden können, z. B. $\mathrm{H}_{2} \mathrm{SO}_{4}, \mathrm{H}_{2} \mathrm{O}$; $\left.\mathrm{H}_{2} \mathrm{SO}_{4}, 2 \mathrm{H}_{2} \mathrm{O}^{1}\right) ; \mathrm{H}_{2} \mathrm{SO}_{4}, 4 \mathrm{H}_{2} \mathrm{O}^{1}$ ) Eine grössere $\mathrm{Zahl} \mathrm{von}$ Hydraten der Schwefelsäure haben Mendelejeff und auch Pickering von den Sprungpunkten abgeleitet, die man erhält, wenn man das Verhältniss der Dichte der wässerigen Lösungen zum Procentgehalt in Rücksicht zieht. Es liegt mir ferne, über den Werth oder Unwerth dieser Methode, die verschiedentlich abfällig kritisirt wurde, hier ein Urtheil abgeben

1) Vgl. die Arbeiten von C. H. Jones, Amer. Chem. J. 16, 1-19, 1894; E. Biron, Journ. russ. chem. Ges. 31, 515, 1899. 
142 Vaubel: Verhältniss zwischen Reactionsvermögen etc.

zu wollen. Immerhin muss zugegeben werden, dass wenigstens einige der "vorhergesagten" Hydrate isolirt werden konnten.

Einige von mir angestellte Versuche haben ergeben, dass bestimmte Reactionen organischer Farbstoffe mit Schwefelsäure höherer Concentration vorerst nur durch Annahme von eigenartigen Combinationen, die durch den grösseren oder geringeren Wassergehalt bedingt sind, erklärt werden können. Ich gebe zunächst folgende Beispiele, die natürlich noch sehr vermehrt werden können.

1) Rosindulin. Wie in den D. R. P. 45370 und 50822 sowie in der sonst noch vorhandenen Litteratur über die Rosindulinfarbstoffe angeführt ist, geben dieselben eine charakteristische grüne Färbung mit concentrirter Schwefelsäure. Bereits vor mehreren Jahren wurde mir Gelegenheit, diese Reaction näher zu untersuchen, und ich fand, dass z. B. Phenylrosindulin erst dann Grünfärbung giebt, wenn der Gehalt der Schwefelsäure nicht mehr als $95,2 \% \mathrm{H}_{2} \mathrm{SO}_{4}$ beträgt. Ist derselbe höher, so tritt Braunfärbung auf, die auf Zusatz von Wasser in Grün umschlägt, sobald obige Verdünnung erreicht ist. Der Uebergangspunkt ist sehr scharf zu erkennen. Eine Schwefelsäure von $95,2 \% \mathrm{H}_{2} \mathrm{SO}_{4}$ entspricht ungefähr dem Verhältniss

$$
18 \mathrm{H}_{2} \mathrm{SO}_{4}: 5 \mathrm{H}_{2} \mathrm{O} \text {. }
$$

Die Leitfähigkeit beginnt hier in etwas rascherem Tempo abzunehmen als vorher.

2) Safranin. Bekanntlich haben die Safraninfarbstoffe die Eigenschaft, je nach der Menge der vorhandenen Säure die Farbe der Lösung zu verändern. Hierüber berichtete bereits R. Nietzkil) in Betreff des Phenosafranins: „Starke Schwefelsäure färbt es grün, etwas verdünntere Schwefelsäure oder concentrirte Schwefelsäure dagegen blau. Bei weiterer Verdünnung erhält man die rothe bezw. rothviolette Farbe der Safraninlösungen. Offenbar beruht dieser Farbenwechsel auf der Existenz von ebenso viel verschiedenen Salzen, welche mit Ausnahme der einsäurigen rothen unbeständig sind. Das eine Säure-Molekül wird dagegen sehr energisch festgehalten,

1) R. Nietzki, Ber. 16, 468, 1883. 
und die einsäuerigen Salze werden selbst durch kaustische Alkalien nicht zersetzt.“

Ich habe mich nun bemüht, die sehr schwierige Frage zu entscheiden, bei welcher Concentration ein Farbenumschlag eintritt. Zum besseren Vergleich habe ich auch noch die Versuch auf Salzsäure ausgedehnt und gebe weiterhin in der folgenden Tabelle noch die elektrischen Leitfäbigkeiten der verschieden concentrirten Säuren, wie sie sich aus den Untersuchungen von Kohlrausch dnrch Interpolation berechnen. Der Säuregehalt ist in Procenten angegeben und ist in der letzten Spalte noch das Verhältniss der Grammäquivalente Schwefelsäure zu denen der Salzsäure angegeben. Bei der Schwierigkeit der Materie sind, da die Beobachtung der feineren Farbenunterschiede nicht absolut genau zu erreichen ist, die Resultate selbstverständlich nur angenäherte.

\begin{tabular}{|c|c|c|c|c|c|}
\hline \multirow[b]{2}{*}{ Farbe } & \multicolumn{2}{|c|}{$\mathrm{H}_{2} \mathrm{SO}_{4}$} & \multicolumn{2}{|c|}{$\mathrm{HCl}$} & \multirow{2}{*}{$\begin{array}{c}\text { Verhältniss- } \\
\text { zahlen der } \\
\text { Grammäqui- } \\
\text { valente }\end{array}$} \\
\hline & $\%$ & $\begin{array}{c}\text { Leitfähig- } \\
\text { keit }\end{array}$ & $\%$ & $\begin{array}{c}\text { Leitfähig- } \\
\text { keit }\end{array}$ & \\
\hline Reinblau & 53,51 & $4550^{10-8}$ & - & - & $1 / 2 \mathrm{H}_{2} \mathrm{SO}_{4}: 1 \mathrm{HCl}$ \\
\hline Blauviolett & 41,82 & $6100^{10-8}$ & 25,41 & $6650^{10-8}$ & $0,854: 0,699$ \\
\hline Blaurothviolett & 37,76 & $6500^{10-8}$ & 21,89 & $6950^{10-8}$ & $0,700: 0,600$ \\
\hline Rothviolett & 24.75 & $6710^{10-8}$ & 16,5 & $6680^{10-8}$ & $0,505: 0,452$ \\
\hline $\begin{array}{l}\text { Neutrallackmus- } \\
\text { farben }\end{array}$ & 18,46 & $5900^{10-8}$ & 14,52 & $6444^{10-8}$ & $0,377: 0,398$ \\
\hline
\end{tabular}

Die Tabelle zeigt, dass mit Abnahme der Concentration auch die Anzahl der zum Farbenwechsel nöthigen Grammäquivalente an Schwefelsäure sich derjenigen der Salzsäure nähern, bis sie zum Schlusse nahezu übereinstimmen. Jedenfalls beweisen aber die Resultate, dass die elektrische Leitfähigkeit der Säurelösung bei der Verwandlung der mehrfachen sauren Salze des Phenosafranins von geringer Bedeutung zu sein scheint, was angesichts der Thatsache, dass die Leitfähigkeit neben der Koncentration auch von der Reibung der Jonen an ihren Nachbarn und am Lösungsmittel, dem elektrolytischen Reibungswiderstand, abhängig ist, nicht wunderbar erscheint, denn dieser letztere scheint mir wiederum neben der einen oder anderen Ursache von dem möglichen $\nabla$ orhandensein dieses oder jenes Hydrates bedingt zu sein. Lösungen mit gleicher 
144 Vaubel: Verbältniss zwischen Reactionsvermögen etc.

Leitfähigkeit zeigen demgemäss nicht die gleiche Wirkung selbst bei derselben Säure.

Hieran schliesst sich noch als weiterer Beweis, wie sehr die Reactionsfähigkeit der Schwefelsäure von dem eventuellen Vorhandensein des einen oder anderen Hydrats abhängig ist, das Verhalten der Schwefelsäure gegen Thiosulfat.

3) Natrium thiosulfat. Wie ich bereits in einer Arbeit "Ueber das Verhalten des Natriumthiosulfats gegen Säuren, insbesondere gegen Schwefelsäure und Salz-

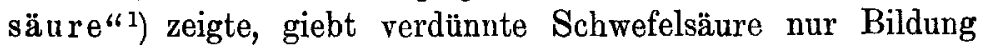
von Schwefeldioxyd und Ausscheidung von Schwefel. Bei Anwendung einer Säure von $84,1 \%$ Schwefelsäure zeigen sich jedoch deutlich erkennbare Mengen von Schwefelwasserstoff, diemit derConcentration der Schwefelsäure immer mehr zunehmen und bei ganz concentrirter Säure sehr stark auftreten. Eine Säure von $84,1 \% \mathrm{H}_{2} \mathrm{SO}_{4}$ entspricht annähernd dem Hydrat $\mathrm{H}_{2} \mathrm{SO}_{4}$, $\mathrm{H}_{2} \mathrm{O}$, wobei noch der Wassergehalt des Thiosulfats zu berücksichtigen is t.

Also auch hier ist wiederum der Einfluss der Hydratbildung unverkennbar, und darf diese Beobachtung, die zu einem bereits bekannten Hydrat führt, als sicherer Beweis für meine Annahme angesehen werden, dass nicht nur die Jonenconcentration, sondern auch die Hydratbildung von grossem Einfluss auf die Reactionsfähigkeit ist. Es giebt auch einige weitere Fälle, und gerade bei dem Verhalten des Natriumthiosulfats, die unzweifelhaft darthun, dass neben der Jonenconcentration auch die Individualität der betreffenden Säuren von ausserordentlicher Bedeutung ist. Vielleicht kann ich auf die Besprechung dieser Verhältnisse in einiger Zeit zurückkommen.

Darmstadt, Technische Hochschule.

1) W. Vaubel, Ber. 22, 1685, 1889. 